\section{Cahiers de Narratologie}

Analyse et théorie narratives

$19 \mid 2010$

Images composites, arts pluriels

\title{
«C'est maintenant du passé » de Marianne Rubinstein
}

\section{Noémi Kila}

\section{(2) OpenEdition}

Journals

Édition électronique

URL : http://journals.openedition.org/narratologie/6229

DOI : 10.4000/narratologie.6229

ISSN : 1765-307X

Éditeur

LIRCES

\section{Référence électronique}

Noémi Kila, « «C'est maintenant du passé » de Marianne Rubinstein », Cahiers de Narratologie [En ligne], 19 | 2010, mis en ligne le 22 décembre 2010, consulté le 03 mai 2019. URL : http:// journals.openedition.org/narratologie/6229; DOI : 10.4000/narratologie.6229

Ce document a été généré automatiquement le 3 mai 2019.

Article L.111-1 du Code de la propriété intellectuelle. 


\title{
"C'est maintenant du passé " de Marianne Rubinstein
}

\author{
Noémi Kila
}

\section{RÉFÉRENCE}

Paris, Gallimard, 161 pages, 2009

«Les mots latins de fragmen, de fragmentum viennent de frango, briser, rompre, fracasser, mettre en pièces, en poudre, en miettes, anéantir. En grec, le fragment, c'est le klasma, l' apoklasma, l'apospasma, le morceau détaché par fracture, l'extrait, quelque chose d'arraché, de tiré violemment. Le spasmos vient de là : convulsion, attaque nerveuse, qui tire, arrache, disloque. »- rappelle Pascal Quignard dans Une gêne technique à l'égard des fragments. L'écriture fragmentaire est particulièrement adaptée à la démarche entreprise par Marianne Rubinstein dans son dernier livre intitulé C'est maintenant du passé. L'auteur part en quête de l'histoire de ses grand-parents disparus dont elle ne connaîtra l'existence que par bribes, par fragments. Rien que le titre du roman indique qu'il s'agit entre autres de la subversion du temps, notamment puisque les différents membres de famille jadis déportés par les nazis ne deviennent présents que grâce et au sein du récitmême. L'explication du titre fournie par le récit-même en préfigure quelques éléments récurrents : «Dans le recueil le plus célèbre de contes traditionnels japonais - le Konjaku monogatari - , l'équivalent de notre Il était une fois se dit: C'est maintenant du passé. Mais alors que notre Il était une fois met le passé à distance, le C'est maintenant du passé japonais (...) entremêle passé et présent, le passé mordant sans cesse sur le présent, et le présent, à peine vécu, devenant passé à son tour. "

2 La fragmentarité et l'hybridité que l'écriture rubinsteinienne induit caractérise non seulement les éléments de l'histoire personnelle et la perception du temps, mais également la technique narrative et la mise en page du récit. L'œuvre intègre non seulement des photos, mais également des cartes postales et des lettres pour (re)travailler 
l'histoire personnelle et celle de la Shoah. On trouve entremêlé dans ce récit des témoignages de ceux qui ont connu la famille paternelle de l'auteur, des arrêtés, des souvenirs personnels sur l'enfance et des anecdotes concernant la période de l'enquêtemême, des lettres privées, ou encore la reproduction d'une facture ainsi que des réflexions sur le processus de l'écriture. On rencontre aussi la référence à un autre univers qui constitue le contre-point essentiel du récit d'enquête : celui de la littérature japonaise, des poèmes et contes traditionnels, des Notes de chevet de Sei Shônagon et des haïkus. Paradoxalement, c'est le silence qui relie le projet littéraire et haïkus japonais : «Utiliser ces entrelacs pour appréhender quelque chose auquel les mots seuls ne donnent pas accès, de plus enfoui que le langage, d'inaccessible à la pensée. (...) Ces haiku (...) m'enseignent qu'il est possible d'écrire sur des fragments, des bribes et des silences. » - lit-on dans un fragment.

3 Quant à sa mise en page, C'est maintenant du passé est construit des bribes de mémoire de 1 à 3 pages, souvent entrecoupées par des reproductions de photos, de carte postales et mises en évidence par des espaces vides, équivalentes des moments de silence. Une certaine correspondance entre les personnes représentées sur les cartes et sur les photos reproduites est possible, mais demande-dans la majorité des cas-un travail de déchiffrement. Les personnes représentées sont soit incarnées, voire remplacées par une image, soit - pour être identifiées - le récit dirige le lecteur vers la découverte des jeux de va et vient entre le texte et l'image. Les références aux détails de la photo qui apparaît soit ultérieurement, soit postérieurement - mais en tout cas en décalage avec le texte - ne font que rendre plus présents les liens entre le texte et l'image. On note également la triple structure de la description - formellement analogue aux haïkus - et le caractère fragmenté des portraits.

4 Pour contrebalancer les effets produits par la mémoire et la mise en page brisée, Rubinstein introduit des "ruses» de type narratologique, typo-et orthographique. Ce sont en fait les interrogations et les idées discrètement évoquées par le narrateur qui relient les différentes bribes de mémoire. Tel est le cas de la question posée en début des deux premières bribes, "Que sait-on d'eux?", ainsi que celui du constat relatif au pourquoi de l'écriture. «J'écris parce que j'ai un problème de place. »- sonne la réponse. Voici également un exemple qui met en avant une orthographie défaillante; l'orthographie d'origine de la grand-mère: "Tu me manque partouts... la nuit de mes reives sont de toi... le jour sons longue sans toi mon chéri... ta femme que pense toujour à toi ", écrivait ma grand-mère dans un français maladroit à son mari detenu en Allemagne. »

5 Ces techniques exploitées reflètent non seulement la logique des thématiques évoquées par le récit, mais en constituent également un élément intertextuel important, une technique narrative adaptée pour combler les vides présents aussi bien dans la mémoire du narrateur que dans la structure du texte: «Dès qu'il a fallu commencer, l'idée d'imaginer, d'inventer, de compléter sur cet événement historique particulier m'a insupporté. (...) À la pince à épiler, j'ai récolté les fragments que je trouvais, maigres et fragiles comme des ailes de papillon. Certains avaient des couleurs flamboyantes (...).»

6 Cette hybridité des supports et de genres, ainsi que les différents jeux qui se dessinent entre les différents fragments (mais également à l'intérieur des bribes) convergent avec l'impossibilité de rendre compte du moment de l'histoire en question. La reconstruction d'une mémoire fragmentée et la découverte d'une esthétique hybride invitent les lecteurs à la découverte d'une œuvre à la fois personnelle et inventive. 


\section{AUTEUR}

NOÉMI KILA

Université de Pécs (Hongrie), Université d'Artois 